\title{
Correction to: A case-control study of the risk of upper gastrointestinal mucosal injuries in patients prescribed concurrent NSAIDs and antithrombotic drugs based on data from the Japanese national claims database of 13 million accumulated patients
}

\author{
Nobuyuki Sugisaki ${ }^{1}$ (D) Ryuichi Iwakiri ${ }^{1} \cdot$ Nanae Tsuruoka $^{2} \cdot$ Yasuhisa Sakata $^{2}$. \\ Ryo Shimoda $^{2}$ - Shun Fujimoto ${ }^{1,2}$ - Yuichiro Eguchi ${ }^{1,2} \cdot$ Kazuma Fujimoto $^{1,2}$ \\ Published online: 19 September 2018 \\ (c) Japanese Society of Gastroenterology 2018
}

\section{Correction to: J Gastroenterol https://doi.org/10. 1007/s00535-018-1483-x}

The authors would like to correct the errors in the publication of the original article. The correction details are given below for your reading.

The first sentence in the "Discussion" section should be "The present case-control study with a large number of patients demonstrated: (i) the risk of peptic ulcers and upper GI bleeding increased by prescribing NSAIDs and/or antithrombotic agents, and the odds ratios with these drugs ranged from 1.31-2.38, which was increased by concurrent use of these drugs; and (ii) prescribing corticosteroids and bisphosphonates, lifestyle-related diseases, and smoking also increased the risk of peptic ulcers and upper GI bleeding.".

In addition, the Tables 2 and 3 were published incorrectly. The corrected Tables 2 and 3 are given in the following pages.

The original article can be found online at https://doi.org/10.1007/ s00535-018-1483-x.

Nobuyuki Sugisaki

nobuyuki_sugisaki@eapharma.co.jp;

n-sugisaki@hhc.eisai.co.jp

1 Graduate School of Medical Science, Saga University, 5-1-1

Nabeshima, Saga 849-8501, Japan

2 Department of Internal Medicine, Saga University, Saga 849-8501, Japan 
Table 2 Odds ratios for the risk of peptic ulcers for each prescribed high-risk drug and lifestyle-related disease

\begin{tabular}{|c|c|c|c|c|c|}
\hline $\begin{array}{l}\text { Concurrent } \\
\text { drugs/complications }\end{array}$ & $\begin{array}{l}\text { Cases } \\
(n=143,271)\end{array}$ & $\begin{array}{l}\text { Controls } \\
(n=1,432,710)\end{array}$ & $\begin{array}{l}\text { Crude odds } \\
\text { ratio }\end{array}$ & $\begin{array}{l}\text { Multivariable odds ratio (95\% } \\
\text { CI) }\end{array}$ & $p$ value \\
\hline NSAIDs & $4501(3.14 \%)$ & $28,696(2.00 \%)$ & 1.56 & $1.45(1.41-1.50)$ & $<0.001$ \\
\hline COX-2 inhibitors & $2254(1.57 \%)$ & $15,969(1.11 \%)$ & 1.42 & $1.31(1.25-1.37)$ & $<0.001$ \\
\hline Low-dose aspirin & $3522(2.46 \%)$ & $21,969(1.53 \%)$ & 1.62 & $1.50(1.44-1.55)$ & $<0.001$ \\
\hline Antiplatelet drugs & $3900(2.72 \%)$ & $22,847(1.60 \%)$ & 1.72 & $1.53(1.48-1.59)$ & $<0.001$ \\
\hline Anticoagulants & $1563(1.09 \%)$ & $9153(0.64 \%)$ & 1.72 & $1.62(1.54-1.71)$ & $<0.001$ \\
\hline Corticosteroids & $15,140(10.57 \%)$ & $120,201(8.39 \%)$ & 1.29 & $1.24(1.22-1.26)$ & $<0.001$ \\
\hline Bisphosphonates & $1858(1.30 \%)$ & $9220(0.64 \%)$ & 2.03 & $1.84(1.75-1.94)$ & $<0.001$ \\
\hline Hypertension & $32,169(22.45 \%)$ & $351,898(24.56 \%)$ & 0.89 & $0.78(0.77-0.79)$ & $<0.001$ \\
\hline Diabetes mellitus & $20,542(14.34 \%)$ & $180,173(12.58 \%)$ & 1.16 & $1.15(1.13-1.17)$ & $<0.001$ \\
\hline Hyperlipidemia & $25,424(17.75 \%)$ & $222,023(15.50 \%)$ & 1.18 & $1.19(1.18-1.21)$ & $<0.001$ \\
\hline Hypercholesterolemia & $12,809(8.94 \%)$ & $124,604(8.70 \%)$ & 1.03 & $1.01(0.99-1.03)$ & 0.575 \\
\hline
\end{tabular}

$n$ number, $C I$ confidence interval, NSAIDs ( $>28$ days in the 3 months before diagnosing GI injury) nonsteroidal anti-inflammatory drugs, GI gastrointestinal

Table 3 Odds ratios for upper gastrointestinal bleeding for each high-risk drug and lifestyle-related disease

\begin{tabular}{|c|c|c|c|c|c|}
\hline $\begin{array}{l}\text { Concurrent } \\
\text { drugs/complications }\end{array}$ & $\begin{array}{l}\text { Cases } \\
(n=10,545)\end{array}$ & $\begin{array}{l}\text { Controls } \\
(n=105,450)\end{array}$ & Crude odds ratio & Multivariable odds ratio $(95 \% \mathrm{CI})$ & $p$ value \\
\hline NSAIDs & $472(4.48 \%)$ & $2250(2.13 \%)$ & 2.15 & $1.76(1.59-1.96)$ & $<0.001$ \\
\hline COX-2 inhibitors & $217(2.06 \%)$ & $1146(1.09 \%)$ & 1.91 & $1.62(1.40-1.88)$ & $<0.001$ \\
\hline Low-dose aspirin & $455(4.31 \%)$ & $1964(1.86 \%)$ & 2.38 & $1.96(1.75-2.19)$ & $<0.001$ \\
\hline Antiplatelet drugs & $469(4.45 \%)$ & $2035(1.93 \%)$ & 2.37 & $1.82(1.63-2.03)$ & $<0.001$ \\
\hline Anticoagulants & $241(2.29 \%)$ & $841(0.80 \%)$ & 2.91 & $2.38(2.05-2.77)$ & $<0.001$ \\
\hline Corticosteroids & $1507(14.29 \%)$ & $8675(8.23 \%)$ & 1.86 & $1.75(1.65-1.86)$ & $<0.001$ \\
\hline Bisphosphonates & $135(1.28 \%)$ & $677(0.64 \%)$ & 2.01 & $1.64(1.35-1.99)$ & $<0.001$ \\
\hline Hypertension & $3055(28.97 \%)$ & $29,017(27.52 \%)$ & 1.07 & $0.96(0.91-1.01)$ & 0.098 \\
\hline Diabetes mellitus & $2099(19.91 \%)$ & $14,937(14.17 \%)$ & 1.51 & $1.56(1.47-1.65)$ & $<0.001$ \\
\hline Hyperlipidemia & $1839(17.44 \%)$ & $17,864(16.94 \%)$ & 1.04 & $0.89(0.84-0.94)$ & $<0.001$ \\
\hline Hypercholesterolemia & $942(8.93 \%)$ & $9806(9.30 \%)$ & 0.96 & $0.81(0.75-0.87)$ & $<0.001$ \\
\hline
\end{tabular}

$n$ number, $C I$ confidence interval, NSAIDs ( $>28$ days in the 3 months before diagnosing GI injury) nonsteroidal anti-inflammatory drugs, GI gastrointestinal 